\title{
Spreading of superfluid vorticity clouds in normal-fluid turbulence
}

\author{
DEMOSTHENES KIVOTIDES $\dagger$ \\ Department of Chemical Engineering, University of California, Santa Barbara, CA 93117, USA
}

(Received 19 May 2010; revised 4 September 2010; accepted 5 September 2010; first published online 16 December 2010)

In this paper, we formulate a self-consistent model of thermal superfluid dynamics. By solving it, we analyse the problem of superfluid vorticity cloud propagation in normal-fluid turbulence. We show that superfluid cloud expansion is driven by pattern-forming superfluid vortex instabilities taking place in the interface layer between the cloud's bulk and the outer undisturbed normal-fluid turbulence. The radius of the cloud increases linearly with time. Mutual friction transfers energy from the normal-fluid turbulence to the superfluid cloud, whilst damping the smallest normal-fluid turbulence motions. This damping action is much weaker than viscous dissipation effects in a corresponding pure normal-fluid turbulence. The energy spectrum of superfluid turbulence presents the $k^{-3}$ scaling that characterizes the spiral superfluid vorticity patterns of normal vortex tube-superfluid vortex interactions. The corresponding $k^{-2}$ pressure spectrum signifies the singular nature of superfluid vorticity. These two scalings coincide in wavenumber space with the Kolmogorov regime in the normal-fluid turbulence. We compute a fractal dimension $d_{f} \approx 1.652$ for superfluid vorticity. Due to simpler underlying superfluid vortex dynamics in relation to the strongly nonlinear classical vortex dynamics, this fractal dimension is smaller than the corresponding dimension of vortex tube centrelines in classical turbulence.

Key words: quantum fluids, turbulent flows, vortex flows

\section{Introduction}

The classical (simple or complex) fluids of everyday experience obey microscopic, Hamiltonian dynamics encoding a fundamental force law (e.g. the Lennard-Jones potential). Since in many cases only an effective large-scale description of such systems is needed, the methods of statistical mechanics have been employed in order to coarse-grain the microscopic dynamics (Gardiner 2004). Thus, the latter have been re-expressed in the form of the exact probabilistic Liouville equation and coarsegrained in order to obtain the Boltzmann equation in the kinetic range of scales. By taking the hydrodynamic limit of the Boltzmann equation, fluid dynamical equations (e.g. the Navier-Stokes) have been derived. Turbulence in fluids requires further coarse-graining of the original dynamics, however, at present, no standard theory of Navier-Stokes statistics is available.

The development of quantum mechanics and the performance of ultra-low temperature experiments motivated similar studies of quantum Hamiltonian systems. Contrary to expectations on the basis of the very different microscopic physics, the 
hydrodynamic phenomenology of such systems is not necessarily non-classical. Indeed, in such systems, quantum and thermal fluctuations coexist and the dominating effects of the latter lead to hydrodynamic behaviour similar to that of classical fluids. One such important example is quark gluon plasma that at hydrodynamic scales behaves like a classical (albeit relativistic) compressible fluid (Wilson \& Mathews 2007). In order to obtain non-classical hydrodynamic behaviour two things are necessary: (a) quantum probabilities are projected on macroscopic scales without decoherence inducing thermal fluctuation effects and $(b)$ the motion of a significant proportion of the total number of particles obeys the same quantum probability function (wave function). The occupation of the same quantum state by many particles ensures that averages based on this state predict the velocity of a fluid made of these particles measured by an actual experimental probe. This is not the case for one quantum particle, since, in this case, an experimental probe measures at each separate experiment a velocity that could differ greatly from the average velocity predicted by the particle's wave function (quantum fluctuations). The physical mechanism that provides the required ingredients and makes the above described superfluid behaviour possible is called Bose-Einstein condensation (Leggett 2006).

Following Bogoliubov and Beliaev, Bose-Einstein condensation is modelled as a phase transition in the quantum dynamics of particle systems (Griffin, Kinuni \& Zaremba 2009). Due to this phase transition, a significant portion of the particles occupy the same quantum state and comprise the superfluid. The non-condensed particles are characterized by both quantum and thermal fluctuations (i.e. are in a mixed quantum state) thus, by resembling a classical fluid, are called normal fluid. The normal fluid and superfluid constituents interact via mutual friction forces. Both fluids can become turbulent (Vinen \& Niemela 2002; Finne et al. 2006). However, due to the very different nature of the statistics obeyed by their constituent particles, the corresponding turbulence physics in the two fluids is different. Indeed, the superfluid obeys Gross-Pitaevskii-type equations that allow the compact formulation of fluid dynamics in terms of a (complex) scalar wavefunction (Pitaevskii \& Stringari 2003). In this formulation, vortices are discrete, potential, quantized and unconditionally stable due to their topological defect nature. Since, at macroscopic scales, their apparent core size is zero, they cannot be stretched and produce a Kolmogorov-type turbulence cascade (Kivotides 2007a,b; Kivotides \& Wilkin 2009). These characteristics are in sharp contrast with the continuous viscous finite-core size vortices of Navier-Stokes turbulence that can be either vortex filaments or sheets and can become unstable and merge with the background fluctuating vorticity field. The intriguing interplay of these two different turbulence phenomenologies as realized via mutual friction force interactions between the superfluid and normal-fluid constituents is called thermal superfluid turbulence and is the topic of the present investigation.

The experimental investigation of thermal superfluid turbulence presents major challenges (Fisher \& Pickett 2008; Skrbek \& Vinen 2008). Our discussion is confined to superfluid ${ }^{4} \mathrm{He}$, which is the reference superfluid in our analysis. Despite many ingenious experimental techniques, the routine unambiguous measurement of the velocities of the two constituent fluids is not feasible at present. For example, the second sound technique is capable of measuring the superfluid vortex line density and has been applied by Stalp, Skrbek \& Donnelly (1999) in homogeneous isotropic superfluid turbulence experiments. In the same flow context, Maurer \& Tabeling (1998) measured pressure fluctuations and made inferences about energy spectra. In more recent developments, Zhang \& Van Sciver (2005) employed particle image velocimetry with polymer microspheres in order to measure the normal-fluid velocity. 
A different method employing particle-tracking capability of solid hydrogen tracers has been applied by Bewley, Lathrop \& Sreenivasan (2006) in the visualization of superfluid vortices. Since the particles are not really passive flow tracers and interact with the flow structure, the interpretation of the experimental data obtained with particle methods is not straightforward (Kivotides, Barenghi \& Sergeev 2008a; Kivotides 2008a,b; Kivotides \& Wilkin 2008). Specially targeted experiments in conjunction with theoretical analysis are needed in order to probe essential thermal superfluid physics. For this reason, the further development and application to turbulent flows of the tracking of metastable helium molecules as developed by Guo et al. (2009) is highly desirable. By allowing the determination of the undisturbed normal-fluid velocity, this approach can boost thermal superfluid turbulence studies.

Similar difficulties exist on the theoretical research side (Vinen 2000; Tsubota \& Kobayashi 2008; Barenghi \& Niemela 2009). Even elementary questions like the scaling of energy spectra are not definitely understood. Part of the difficulty has to do with the complexity of the underlying physics and the disparate ranges of scales involved. In this work, we contribute to theoretical superfluid turbulence from two points of view: $(a)$ we focus on the thermal superfluid analogue of a basic problem in classical turbulence, i.e. the spreading of turbulent clouds and $(b)$ we formulate a selfconsistent model of the corresponding incompressible thermal superfluid dynamics and solve it by using numerical and computational methods. Regarding point $(a)$, we prepare a homogeneous isotropic normal-fluid turbulence in steady state and insert in it a seed superfluid vorticity. Due to mutual friction excitation from the energetic normal fluid, the latter grows forming an expanding cloud. We analyse the geometrical and dynamical characteristics of this cloud and connect them with basic physical processes. Certainly, many existing experimental methods could be applied to this problem in order to measure many of the quantities computed here from first principles. Regarding point $(b)$, we note that our analysis is applicable only in the hydrodynamic range of scales. Indeed, in analogy with classical fluids, investigations in the kinetic regime of space-time scales are based on the quantum Boltzmann equation (Jackson, Barenghi \& Proukakis 2007; Griffin et al. 2009), and truly microscopic studies are based on quantum molecular dynamics as the latter are encoded in the formalism of quantum field theory (Mine et al. 2007). Finally, a further coarse-graining of our model would correspond to theories of the HallVinen (Hall \& Vinen 1956) and Bekharevich-Khalatnikov (Leggett 2006) type, i.e. to statistical theories of thermal superfluid turbulence (Henderson \& Barenghi 2004; Peralta et al. 2005, 2006, 2008; Andersson, Sidery \& Comer 2007; Sidery, Andersson \& Comer 2008; Melatos \& Peralta 2010). The mathematical difficulties involved in this coarse-graining are great, and our results could guide the relevant analyses.

\section{Model of thermal superfluid dynamics}

In general, fluid dynamics can be described in terms of the following: $(a)$ conservation laws (mass, energy and momentum), $(b)$ vortex dynamics (vorticity and enstrophy) and (c) wavefunctions or streamfunctions (scalar or vector fields). The wavefunction formulation (in terms of a complex scalar field) of superfluid dynamics is elegant and powerful. Yet, when addressing large-scale flows, it is more efficient to employ a vortex dynamical formulation, especially since (as discussed above) the vorticity field in superfluids is discrete. On the other hand, the conservation law viewpoint of normal-fluid dynamics has the advantage of making directly available 
many techniques of analysis (e.g. finite volume methods) that have been developed in the context of classical fluids.

Accordingly, an incompressible thermal superfluid $\mathscr{F}=\left\{\boldsymbol{V}_{n}, \boldsymbol{X}_{s}\right\}$ is specified by the normal-fluid velocity field $\boldsymbol{V}_{n}(\boldsymbol{x}, t)$, and the superfluid vortex field $\boldsymbol{X}_{s}(\boldsymbol{x}, t)$ which is the union $\boldsymbol{X}_{s}(\boldsymbol{x}, t)=\bigcup_{i=1}^{N_{l}(t)} \boldsymbol{X}_{s}^{i}(\xi, t)$ of the contours of $N_{l}(t)$ superfluid line vortices that comprise a vortex link or tangle $\mathscr{L}$. Due to topology-changing vortex reconnections, $N_{l}$ is varying with time. Here, $\boldsymbol{x}$ denotes a point in space, $\xi$ is the arclength parameterization along the vortices and $t$ is time.

Our thermal superfluid dynamics model includes the following six input parameters: (i) superfluid mass density $\rho_{s}$, (ii) normal-fluid mass density $\rho_{n}\left(\rho=\rho_{s}+\rho_{n}\right.$ is the total mass density), (iii) normal-fluid dynamic viscosity $\mu$, (iv) quantum of circulation $\kappa$, (v) longitudinal mutual friction drag coefficient $d_{\ell}$ and (vi) transverse mutual friction drag coefficient $d_{t}$. The first three are temperature dependent material properties, and $\kappa=h / m$ (where $h$ is Planck's constant and $m$ is the mass of the molecules of the thermal superfluid). As shown by Barenghi, Donnelly \& Vinen (1983), the microscopic mutual friction coefficients $d_{\ell}$ and $d_{t}$ can be expressed in terms of the macroscopic experimentally measured mutual friction coefficients of Hall \& Vinen (1956). By specifying appropriate initial and boundary conditions, we evolve $\boldsymbol{V}_{n}(\boldsymbol{x}, t)$ and $\boldsymbol{X}_{s}(\boldsymbol{x}, t)$ according to nonlinear integro-differential laws that we discuss next.

\subsection{Superfluid dynamics}

With respect to the superfluid vortices, the fast, low-amplitude oscillations of their core are not dynamically important in the macroscopic range of scales of interest to us here, and therefore, their inertia is neglected. Accordingly, their governing equation is

$$
-f_{M}=f_{d}+f_{I}
$$

On the left-hand side, $\boldsymbol{f}_{M}$ is the superfluid Magnus or Kutta-Joukowski hydrodynamic lift force per unit length

$$
\boldsymbol{f}_{M}=-\rho_{s} \kappa \boldsymbol{X}_{s}^{\prime} \times\left(\boldsymbol{V}_{s}-\dot{\boldsymbol{X}}_{s}\right)
$$

where $\boldsymbol{V}_{s}$ is the Biot-Savart velocity field

$$
\boldsymbol{V}_{s}\left(\boldsymbol{X}_{s}\right)=\frac{\kappa}{4 \pi} \int_{\mathscr{L}} \frac{\left(\boldsymbol{x}-\boldsymbol{X}_{s}\right) \times \mathrm{d} \boldsymbol{x}}{\left|\boldsymbol{x}-\boldsymbol{X}_{s}\right|^{3}} .
$$

Here, we denote with $\boldsymbol{X}_{s}^{\prime}=\partial \boldsymbol{X}_{s} / \partial \xi$ the unit tangent vector along the superfluid vortices and by $\dot{\boldsymbol{X}}_{s}=\partial \boldsymbol{X}_{s} / \partial t$ their velocity.

The sum of forces on the right-hand side represent the nonlinear coupling between $\boldsymbol{V}_{n}(\boldsymbol{x}, t)$ and $\boldsymbol{X}_{s}(\boldsymbol{x}, t)$, i.e. the mutual friction force. Physically, this force is due to the scattering of normal-fluid phonons (i.e. quantum field fluctuations) from a moving superfluid vortex. In general, the mutual friction force has a transverse and a longitudinal component. Assuming no relative motion between a superfluid vortex and the condensate, Sonin (1997) has shown that the transverse component has two contributions: one from standard scattering theory (that is not valid for very small scattering angles and is associated with the coefficient $d_{t}$ above) and one from a special analysis valid for very small scattering angles that results in the Iordanskii force. In detail, the drag force per unit length based on standard scattering theory is

$$
\boldsymbol{f}_{d}=d_{t} \boldsymbol{X}_{s}^{\prime} \times\left(\boldsymbol{V}_{n}-\dot{\boldsymbol{X}}_{s}\right)-d_{\ell} \boldsymbol{X}_{s}^{\prime} \times\left[\boldsymbol{X}_{s}^{\prime} \times\left(\boldsymbol{V}_{n}-\dot{\boldsymbol{X}}_{s}\right)\right],
$$


and the Iordanskii force per unit length is

$$
\boldsymbol{f}_{I}=-\rho_{n} \kappa \boldsymbol{X}_{s}^{\prime} \times\left(\boldsymbol{V}_{n}-\dot{\boldsymbol{X}}_{s}\right) .
$$

Notably, in their pioneering contributions, Hall \& Vinen (1956) have written an equation for the coarse-grained form of the mutual friction force including their famous experimentally measured transverse and a longitudinal coefficients. Accordingly, the transverse Hall and Vinen coefficient is related to the sum $d_{t}-\rho_{n} \kappa$. There has been some controversy regarding the Iordanskii force. Based on earlier work of Thouless, Ao \& Niu (1996), Wexler has argued that it is identically zero (Wexler 1997). However, Stone (2000) has extended Sonin's analysis in cases for which the superfluid vortex moves with respect to the condensate reaffirming the existence of the Iordanskii force, as well its interpretation by Volovik (1998) as an analogue of the gravitational Bohm-Aharonov effect.

Idowu et al. (2000) have produced a special analytical solution of (2.1) by enforcing a zero vortex velocity along the tangent. After minor rearrangement their equation can be written as

$$
\dot{\boldsymbol{X}}_{s}=\boldsymbol{V}_{s}+h_{1} \boldsymbol{X}_{s}^{\prime} \times\left(\boldsymbol{V}_{n}-\boldsymbol{V}_{s}\right)+h_{2} \boldsymbol{X}_{s}^{\prime} \times\left[\boldsymbol{X}_{s}^{\prime} \times\left(\boldsymbol{V}_{n}-\boldsymbol{V}_{s}\right)\right],
$$

where the dimensionless coefficients $h_{1}$ and $h_{2}$ are

$$
\begin{aligned}
& h_{1}=\frac{\rho_{s} \kappa d_{\ell}}{d_{\ell}^{2}+\left(\rho_{n} \kappa+\rho_{s} \kappa-d_{t}\right)^{2}}, \\
& h_{2}=\frac{\left(d_{t}-\rho_{n} \kappa\right)\left(\rho_{n} \kappa+\rho_{s} \kappa-d_{t}\right)-d_{\ell}^{2}}{d_{\ell}^{2}+\left(\rho_{n} \kappa+\rho_{s} \kappa-d_{t}\right)^{2}} .
\end{aligned}
$$

Equation (2.6) is not an adequate description of superfluid vortex physics, since, in the incompressible limit of Gross-Pitaevskii fluid dynamics, the very important process of superfluid vortex reconnection is lost. This is because reconnections take place at the scale of the vortex core and involve compressibility effects, whilst (2.6) models singular and incompressible vortices. Schwarz developed an effective procedure (surgery) for dealing with topological changes in the superfluid vortex tangle (Schwarz 1995). His approach is supported by the microscopic computation of superfluid vortex reconnection by Koplik \& Levine (1993). In the context of our model, we can summarize his approach as follows: let $\left(\boldsymbol{X}_{s}^{i-1}, \boldsymbol{X}_{s}^{i}, \boldsymbol{X}_{s}^{i+1}\right)$ and $\left(\boldsymbol{X}_{s}^{j-1}, \boldsymbol{X}_{s}^{j}, \boldsymbol{X}_{s}^{j+1}\right)$ be two sequences of points along any superfluid vortex $(i-1 \neq i \neq i+1 \neq j-1 \neq j \neq j+$ 1 ), where increasing $i$ or $j$ indices follow the direction of vorticity. In numerical calculations, the points are separated by the grid size along the vortices (at most half of the effective cutoff scale $\theta$ of $\boldsymbol{X}_{s}$ fluctuations), i.e. $\left|\boldsymbol{X}_{s}^{i}-\boldsymbol{X}_{s}^{i-1}\right|=\theta / 2$. A heuristic analysis of the physics determining $\theta$ is given in Samuels \& Kivotides (1999). In addition, let us define the intervortex spacing scale $\delta=\sqrt{\mathscr{V}_{s} / L}=\lambda^{-1 / 2}$, where $\mathscr{V}_{s}$ is the volume of the space domain encapsulating superfluid vortex tangle, $\mathscr{L}, L$ is the length of $\mathscr{L}$ and $\lambda=L / \mathscr{V}_{s}$ is the vortex line density. Accordingly, our version of Schwarz surgery becomes

$$
\begin{aligned}
&\left(\boldsymbol{X}_{s}^{i-1}, \boldsymbol{X}_{s}^{i}, \boldsymbol{X}_{s}^{i+1}\right) \wedge\left(\boldsymbol{X}_{s}^{j-1}, \boldsymbol{X}_{s}^{j}, \boldsymbol{X}_{s}^{j+1}\right) \wedge\left|\boldsymbol{X}_{s}^{i}-\boldsymbol{X}_{s}^{j}\right|<\min (\theta, \delta) \\
& \longrightarrow\left(\boldsymbol{X}_{s}^{i-1}, \boldsymbol{X}_{s}^{i}, \boldsymbol{X}_{s}^{j+1}\right) \wedge\left(\boldsymbol{X}_{s}^{j-1}, \boldsymbol{X}_{s}^{j}, \boldsymbol{X}_{s}^{i+1}\right) .
\end{aligned}
$$

Notably, when $\theta \rightarrow 0$, we obtain the ideal case where vortices reconnect upon quasi-pointwise contact and vortex length is conserved. However, this ideal vortex length conservation during reconnection comes together with discontinuities in flow 
kinetic energy and vortex curvature. Indeed, Schwarz surgery is better perceived as a coarse-grained model of Gross-Pitaevskii dynamics that assumes a scale separation between the incompressible flow modes resolved in our model and the compressible reconnection processes. Consequently, Schwarz surgery acts on a smooth prereconnection vortex geometry and, without resolving reconnection physics, provides a smooth post-reconnection geometry and a new tangle topology. Since GrossPitaevskii fluid dynamics studies have shown that reconnections transform some of the kinetic flow energy into acoustic energy (Parker et al. 2004), one expects that Schwarz surgery would, in effect, be a dissipative process at large incompressible scales. For the particular surgery employed here, this was shown to be the case, since Kivotides, Sergeev \& Barenghi $(2008 b)$ had to force the superfluid vortex tangle in order to achieve a state with statistically constant length, $L$, at temperature $T=0 \mathrm{~K}$. Overall, Schwarz surgery is a heuristic model of refined microscopic physics. The same holds for its application in the formulation of the vortex tube model of classical incompressible turbulence, where it models subgrid viscous reconnection physics (Kivotides \& Leonard 2003). Notably, in our approach, once the mesh size along the vortices is chosen (i.e. $\theta$ also), Schwarz surgery is fully determined, thus, there are no adjustable parameters.

\subsection{Normal-fluid dynamics}

The normal fluid obeys the forced Navier-Stokes equations

$$
\begin{gathered}
\nabla \cdot \boldsymbol{V}_{n}=0, \\
\frac{\partial \boldsymbol{V}_{n}(\boldsymbol{x}, t)}{\partial t}+\nabla\left(\frac{p}{\rho_{n}+\rho_{s}}+\frac{\boldsymbol{V}_{n} \cdot \boldsymbol{V}_{n}}{2}\right)-\boldsymbol{V}_{n} \times\left(\nabla \times \boldsymbol{V}_{n}\right)-\frac{\mu}{\rho_{n}} \nabla^{2} \boldsymbol{V}_{n} \\
=\frac{\left(\rho_{n} \kappa-d_{t}\right)}{\rho_{n}} \int_{\mathscr{L}} \mathrm{d} \xi\left[\boldsymbol{X}_{s}^{\prime} \times\left(\boldsymbol{V}_{n}-\dot{\boldsymbol{X}}_{s}\right)\right] \delta\left(\boldsymbol{x}-\boldsymbol{X}_{s}\right) \\
+\frac{d_{\ell}}{\rho_{n}} \int_{\mathscr{L}} \mathrm{d} \xi\left\{\boldsymbol{X}_{s}^{\prime} \times\left[\boldsymbol{X}_{s}^{\prime} \times\left(\boldsymbol{V}_{n}-\dot{\boldsymbol{X}}_{s}\right)\right]\right\} \delta\left(\boldsymbol{x}-\boldsymbol{X}_{s}\right)+\left(\epsilon / 3 V_{n}^{2}\right) \boldsymbol{V}_{n},
\end{gathered}
$$

where $p(\boldsymbol{x}, t)$ signifies the scalar pressure field that enforces incompressibility, $\epsilon=-v\left\langle\boldsymbol{V}_{n} \cdot \nabla^{2} \boldsymbol{V}_{n}\right\rangle$ is the rate of kinetic energy dissipation and $V_{n}^{2}=\left\langle\boldsymbol{V}_{n} \cdot \boldsymbol{V}_{n}\right\rangle / 3$ is the mean square of turbulence fluctuations. Here, $v=\mu / \rho_{n}$ is the kinematic viscosity in the normal fluid.

The left-hand side of (2.11) includes the familiar classical fluid dynamics. The first-two terms on the right-hand side model the nonlinear coupling of the normal fluid with the superfluid, thus they are the exact opposites of the right-hand side of (2.1). The three-dimensional delta-functions indicate that the normal fluid is only forced along the quantized vortices. The last term is an artificial external forcing that is tuned to achieve a steady-state homogeneous isotropic normal-fluid turbulence in the absence of the superfluid. The particular form of this force was suggested by Lundgren (2003).

\subsection{Dimensionless numbers}

Overall, given six input parameters, i.e. superfluid mass density $\rho_{s}$, normal-fluid mass density $\rho_{n}$, quantum of circulation $\kappa$, normal-fluid dynamic viscosity $\mu$, longitudinal mutual friction drag coefficient $d_{\ell}$ and transverse mutual friction drag coefficient $d_{t}$, the system of (2.6)-(2.11) provides a complete physical model of incompressible 
thermal superfluid dynamics. Effort is needed in order to understand the physics contained in these equations via various methods of analysis.

Notably, the model differs from the self-consistent model of Kivotides, Barenghi \& Samuels (2001a) in that it uses the correct microscopic mutual friction coefficients $h_{1}$ and $h_{2}$ in (2.6). The coefficients employed in Kivotides et al. (2001a) are not appropriate, since they correspond to coarse-grained superfluid vorticity dynamics that our model aims to resolve via the explicit tracking of individual superfluid vortices. Evidently, incompressible thermal superfluid dynamics is much more complicated than their classical counterpart. Indeed, the latter is fully parameterized by a single dimensionless number (i.e. the Reynolds number), while the former requires four. In particular, by using a typical normal-fluid velocity fluctuation value, $V_{o}$, and a typical normal-fluid length scale, $l_{o}$, one can define a normal-fluid time scale $t_{o}=l_{o} / V_{o}$ and form the following dimensionless numbers: (a) $K=\rho_{n} \gamma / \rho_{s} \kappa$ (where $\gamma=V_{o} l_{o}$ is the circulation of the large normal-fluid eddies) which denotes the relative strength of normal-fluid and superfluid inertial processes, $(b)$ the Reynolds number $\operatorname{Re}=\gamma \rho_{n} / \mu$ which indicates the relative strength of inertial and viscous processes in the normal fluid, (c) $M_{t}=\gamma \rho_{n} /\left|d_{t}-\rho_{n} \kappa\right|$ which marks the relative strengths of inertial and transverse mutual friction effects in the normal fluid and (d) $M_{\ell}=\gamma \rho_{n} / d_{\ell}$ which encodes the relative strengths of inertial and longitudinal mutual friction effects in the normal fluid. Notably, the combinations $M_{t} K^{-1}$ and $M_{\ell} K^{-1}$ gauge the strength of superfluid inertia against mutual friction effects.

As discussed above, routine accurate measurements of normal-fluid or superfluid velocities are not feasible with present experimental capabilities. Thus, the obvious question follows: how do we know that the above complicated model is empirically useful in a quantitative rather than purely qualitative sense? In this respect, we note that although Zhang \& Van Sciver (2005) did not measure the normal-fluid velocity in their experiment, they did measure the velocity of suspended particles in thermal counterflow. The latter was also computed by Kivotides (2008b) who directly solved the present model resolving in a self-consistent manner particle superfluid vortex collisions. Despite the complexity of the underlying physics, the agreement between experiment and theory was excellent, providing support for the empirical usefulness of the present mathematical model. Certainly, future similar comparative studies are highly desirable.

\section{Methods}

The above complex set of equations can only be solved approximately. Its analysis presents a number of numerical and computational challenges having to do with the required combination of vortex methods for the superfluid and finite volume methods for the normal fluid, as well as with purely informatics issues like, for example, the data structures needed in order to code the computational geometry of reconnecting lines in the three-dimensional space. Detailed discussions of the employed methods can be found in Kivotides, Barenghi \& Sergeev $(2008 a, b)$, Kivotides \& Wilkin (2008), Kivotides (2007a) and works cited there. Here, we discuss only the numerical aspect that differentiates normal-fluid dynamics from the familiar classical fluid dynamics: the pointwise mutual friction forcings. In a numerical computation, a discretized version of the three-dimensional delta function is required. Assuming that at time $t$ there are $N_{v}(t)$ differential vortex elements, and denoting by $\boldsymbol{f}_{m f}^{j}, j=1,2$ the mutual friction forces per unit vortex length, the mutual friction integrals in the 
Navier-Stokes equation are discretized as

$$
\sum_{i=1}^{N_{v}} \delta \xi f_{m f}^{j} G\left(x-X_{s}\right),
$$

where the regularization of the delta function is achieved via the Heaviside function $G(\boldsymbol{y})$

$$
G(\boldsymbol{y})=\left\{\begin{array}{lll}
1 / \zeta^{3}, & \left|y_{i}\right|<\zeta / 2, & i=1, \ldots, 3, \\
0, & \left|y_{i}\right|>\zeta / 2, & i=1, \ldots, 3 .
\end{array}\right.
$$

The length scale $\zeta$ ought to be smaller than the cutoff scale $\eta$ of $\boldsymbol{V}_{n}$ fluctuations, the cutoff scale $\theta$ of $\boldsymbol{X}_{s}$ fluctuations, and the intervortex spacing scale $\delta$, i.e. $\zeta<\min (\eta, \theta, \delta)$. This regularization choice implies that each of the $N_{v}$ elements is counted only once when $\zeta$ is chosen to be equal to the normal-fluid grid size $\delta x$. Notably, the regularized normal-fluid equations lose their predictive power beyond the $\zeta$ scale.

\section{Results}

The working fluid is superfluid ${ }^{4} \mathrm{He}$ at temperature $T=1.3 \mathrm{~K}$. Accordingly, the six input parameters are as follows: $\rho_{s}=0.1386 \mathrm{~g} \mathrm{~cm}^{-3}, \rho_{n}=0.00652 \mathrm{~g} \mathrm{~cm}^{-3}, \kappa=$ $9.97 \times 10^{-4} \mathrm{~cm}^{2} \mathrm{~s}^{-1}, \quad \mu=15.2 \times 10^{-6} \mathrm{~g} \mathrm{~cm}^{-1} \mathrm{~s}^{-1} \quad\left(v=2.33 \times 10^{-3} \mathrm{~cm}^{2} \mathrm{~s}^{-1}\right), d_{\ell}=4.27 \times$ $10^{-2} \mathrm{~g} \mathrm{~cm}^{-1} \mathrm{~s}^{-1}$ and $d_{t}=-2.044 \times 10^{-2} \mathrm{~g} \mathrm{~cm}^{-1} \mathrm{~s}^{-1}\left(d_{t}-\rho_{n} \kappa=-2.045 \times 10^{-2} \mathrm{~g} \mathrm{~cm}^{-1} \mathrm{~s}^{-1}\right)$. Accordingly, $M_{t} K^{-1}=6.757 \times 10^{-3}$ and $M_{\ell} K^{-1}=3.236 \times 10^{-3}$. Thus, superfluid dynamics are dominated by mutual friction effects. The computational domain is a periodic cube of size $l_{b}=0.1 \mathrm{~cm}$. Within this domain, we set-up a steadystate homogeneous isotropic normal-fluid turbulence. The turbulence Reynolds number is $R e=V_{n} l_{b} \rho_{n} / \mu=1224$, thus $V_{n}=28.52 \mathrm{~cm} \mathrm{~s}^{-1}$. Employing the relation $\lambda / l_{b}=\sqrt{15} R e^{-1 / 2}$, where $\lambda$ is the Taylor microscale, one finds $R e_{\lambda}=135.5$. Accordingly, $\quad \gamma=V_{n} l_{b}=2.852 \mathrm{~cm}^{2} \mathrm{~s}^{-1}, \quad K=134.567, \quad M_{t}=0.909$ and $M_{\ell}=0.435$. In this way, normal-fluid inertial effects are much stronger than their superfluid counterparts. However, along the superfluid vortex contours, they have magnitude similar to the mutual friction forces, thus the latter are expected to cause a significant transfer of energy from the normal fluid to the superfluid. In order to achieve a steady turbulence state, we start with a white-noise incompressible velocity field as initial condition in the forced Navier-Stokes equation for the normal fluid (Wilkin, Barenghi \& Shukurov 2007). By evolving the initial condition over dozens of large eddy turnover times, we obtain a steady-state Kolmogorov spectrum (figure 1). Subsequently, we insert at the centre of our flow domain a superfluid ring of radius $0.035 l_{b}$, and compute the formation and expansion of the superfluid vorticity cloud. We stop the computation before the cloud reaches the periodic cell boundaries and starts interacting with itself.

For the normal fluid, we employ an $128^{3}$ computational mesh, i.e. the grid size is $\delta x=0.781 \times 10^{-3} \mathrm{~cm}$ which corresponds to wavenumber $k_{\delta x}=1280$. By employing the scaling relation $\eta \approx l_{b} / R e^{3 / 4}$, we find $\eta \approx 0.483 \times 10^{-3} \mathrm{~cm}$ for the Kolmogorov scale. Indeed, as indicated by the spectrum of figure 1 , our flow is adequately resolved. Our delta-function regularization scale is $\zeta=\delta x$. Since, $\zeta$ ought to be smaller than $\theta$, the superfluid vortices are discretized with $\delta \xi=2 \delta x(\theta=2 \delta \xi)$. Moreover, since the smallest intervortex spacing scale $\delta$ recorded in our computations is $\delta=1.23 \times 10^{-3} \mathrm{~cm}$, $\zeta$ satisfies the requirement of being smaller than the actual $\eta, \theta$ and $\delta$ scales in the 
(a)

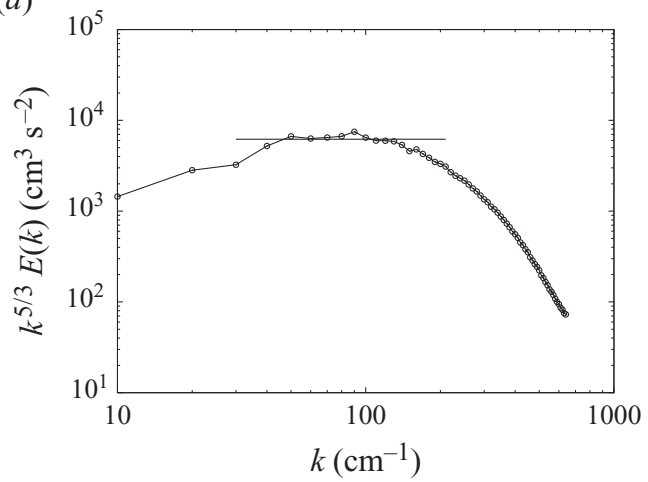

(b)

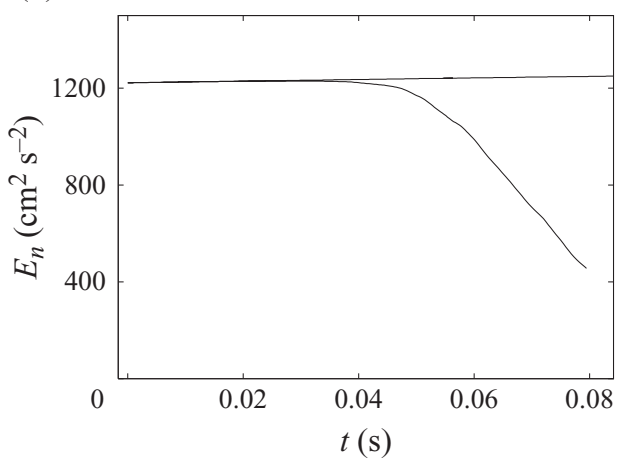

FIGURE 1. (a) A typical (compensated) energy spectrum of the steady-state homogeneous isotropic turbulence in the normal fluid that is employed as initial condition. A Kolmogorov plateau over approximately 100 wavenumbers is evident. The grid size, $\delta x$, of our numerical method corresponds to wavenumber $k_{\delta x}=1280$. (b) Normal-fluid kinetic energy, $E_{n}$, versus time, $t$. The straight horizontal line shows $E_{n}$ for an accompanying computation, where the superfluid is not allowed to react back on the normal fluid.

computations. As the superfluid vortex tangle grows in time, so too does the number of discrete vortex segments $N_{v}$. At the final time, $N_{v} \approx 5 \times 10^{4}$. The time step $\delta t$ is chosen so that the fastest resolved Kelvin waves do not propagate over more than one $\delta \xi$ within $\delta t$, and normal-fluid particles do not propagate more than $1.7 \delta x$ within the same time period (Courant-Friedrichs-Lewy-type condition). The value $\delta t=0.493 \times 10^{-5} \mathrm{~s}$ satisfies both requirements.

\subsection{Normal-fluid energy dynamics}

As shown on figure $1(b)$, the growth of the superfluid vortex cloud is accompanied by a reduction of the normal-fluid kinetic energy per unit mass $E_{n}=(1 / 2 \mathscr{V}) \int \boldsymbol{V}_{n} \cdot \boldsymbol{V}_{n} \mathrm{~d}^{3} \boldsymbol{x}$, where $\mathscr{V}=l_{b}^{3}$. For comparison, the horizontal line shows $E_{n}$ for an accompanying computation, where the superfluid is not allowed to react back on the normal-fluid. As expected, in the latter case, $E_{n}$ is constant in time. Notably, due to numerical truncation and finite arithmetic round-off errors, the constancy of $E_{n}$ is not exact, but there is a $2.4 \% E_{n}$ change in the course of the computation. However, this change is orders of magnitude smaller than the mutual-friction-induced energy transfer between the two fluids, thus it does not affect our conclusions. According to figure $1(b), E_{n}$ decays (to a very good approximation) linearly in time. By defining $D_{s v}=(1 / \mathscr{V}) \int \epsilon_{s v} \mathrm{~d}^{3} \boldsymbol{x}$, we can write

$$
\frac{\partial E_{n}}{\partial t}=-D_{s v}
$$

where $D_{s v}$ can be thought of as the rate of effective dissipation of $E_{n}$ due to an effective 'superfluid vortex viscosity' $v_{s v}$, i.e. $\epsilon_{s v}=-v_{s v}\left\langle\boldsymbol{V}_{n} \cdot \nabla^{2} \boldsymbol{V}_{n}\right\rangle$. This is because our linear forcing counterbalances by default the classical viscous dissipation, thus, in our flow, mutual friction acts as an effective dissipation mechanism that instead of heating up the normal fluid and increasing the energy of molecular fluctuations, augments the complexity, length and energy of the superfluid vortex tangle. We calculate $D_{s v}=2.75 \times 10^{4} \mathrm{~cm}^{2} \mathrm{~s}^{-3}$ and $D=(1 / \mathscr{V}) \int \epsilon \mathrm{d}^{3} \boldsymbol{x}=1.5175 \times 10^{6} \mathrm{~cm}^{2} \mathrm{~s}^{-3}$ for the steadystate turbulence in the normal fluid that was used as initial condition. Thus, it follows that $v_{s v}=D_{s v} v / D \approx 0.02 v$. Evidently, mutual friction forces transfer energy from the normal fluid to the superfluid at a much slower rate than viscous forces would have 
(a)

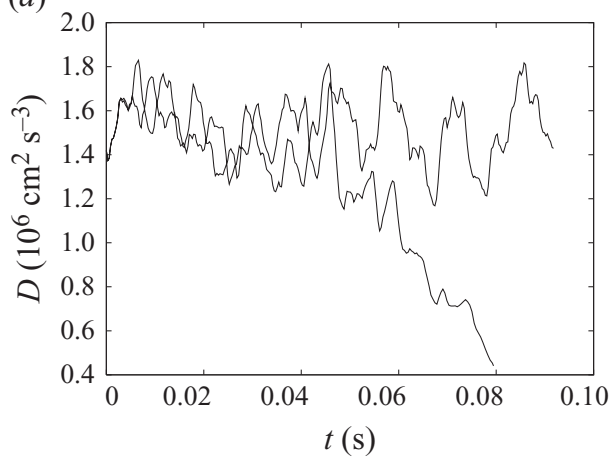

(b)

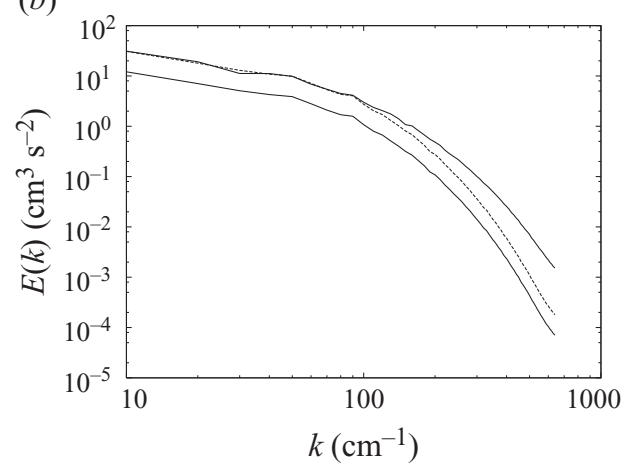

Figure 2. (a) The rate of normal-fluid kinetic energy, $E_{n}$, dissipation due to viscous effects, $D$, versus time, $t$. The $D$ values in an accompanying computation where the superfluid is not reacting back on the normal-fluid are also shown (fluctuating, horizontal line). In both computations, $D$ is compensated by Lundgren's linear forcing. (b) Normal-fluid spectra at initial (upper line) and final times (lower line). After multiplying the final time spectrum with an appropriate factor, we superpose it on the initial one (dashed line). Evidently, mutual friction force acts mostly on the smallest normal-fluid scales. Indeed, not only the high $k$ part of the energy spectrum becomes steeper whilst the low $k$ part retains its slope, but the smaller values of $D$ in the self-consistent case indicate that mutual friction ought to mainly affect small-scale structure, where most of kinetic energy dissipation takes place. Accordingly, for the time duration of our computation, the Kolmogorov inertial regime in the normal-fluid remains intact.

dissipated kinetic energy in a corresponding decaying pure normal-fluid turbulence. This relative weakness of mutual friction has to do with the fact that (unlike viscous forces) it acts only along the superfluid vortices. Next, we show that the effect of $v_{s v}$ is to damp the small scales in the normal-fluid turbulence. Indeed, figure 2(a), compares $D$ in the self-consistent turbulence evolution with $D$ in the steady-state normal-fluid turbulence of an accompanying kinematic computation. Evidently, the $D$ of the self-consistent evolution is decaying in time, which indicates that the cloud grows by damping small flow scales since they are mainly responsible for viscous energy dissipation. A further proof of this is found in figure $2(b)$ which shows the normal-fluid energy spectra at initial (upper line) and final times (lower line). After multiplying the final time spectrum with an appropriate factor, we superpose it on the initial one (dashed line). In agreement with our above conclusion, we see that as time progresses, the high $k$ part of the energy spectrum becomes steeper while the low $k$ part retains its slope, thus mutual friction action alters especially the small-scale structure. Moreover, figure 1(b) indicates that, at final time $t_{f}, \Delta E_{n}=E_{0}-E_{t_{f}}=766.2 \mathrm{~cm}^{2} \mathrm{~s}^{-2}$, i.e. the normal fluid has lost energy $\rho_{n} \Delta E_{n} \mathscr{V}=4.995 \times 10^{-3} \mathrm{~g} \mathrm{~cm}^{2} \mathrm{~s}^{-2}$ to the superfluid. Since the initial superfluid energy is negligible and viscous dissipation is compensated by our linear forcing, $\rho_{n} \Delta E_{n} \mathscr{V}$ must be the superfluid energy at $t_{f}$. Consequently, since the final time superfluid tangle length is $L_{t_{f}}=77.671 \mathrm{~cm}$, the energy per unit vortex length is $\mathscr{E}_{t_{f}}=6.431 \times 10^{-5} \mathrm{~g} \mathrm{~cm} \mathrm{~s}^{-2}$. On the other hand, employing the formula of energy per unit vortex length of a single vortex line threading the centre of a cylinder of radius $l_{b}, \mathscr{E}=\left(\rho_{s} \kappa^{2} / 4 \pi\right) \ln \left(l_{b} / a\right)$, where $a=1.0 \times 10^{-8} \mathrm{~cm}$ is the vortex core size, we find $\mathscr{E}=1.768 \times 10^{-5} \mathrm{~g} \mathrm{~cm} \mathrm{~s}^{-2}$. The difference in the $\mathscr{E}$ values could indicate the tendency of superfluid line vortices to become aligned in our flow, since the total energy (per unit length) of two aligned vortices is greater than the sum of their 
(a)

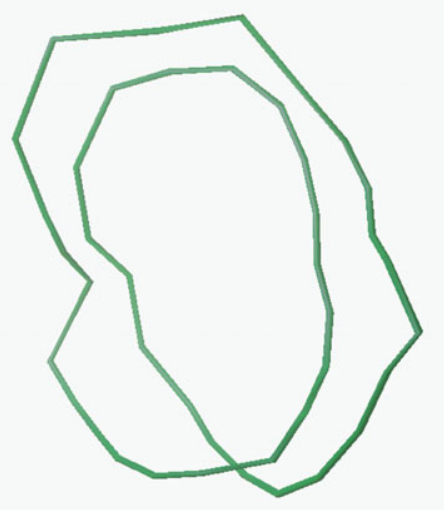

(c)

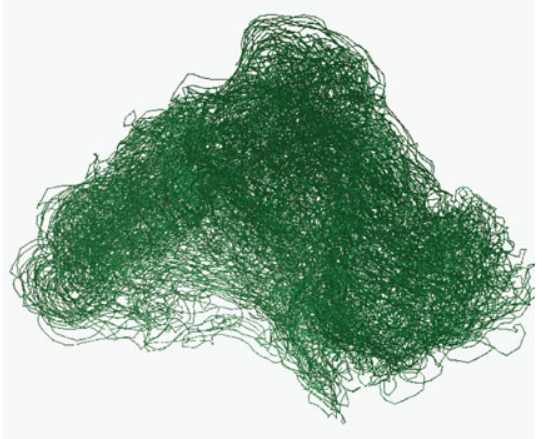

(b)

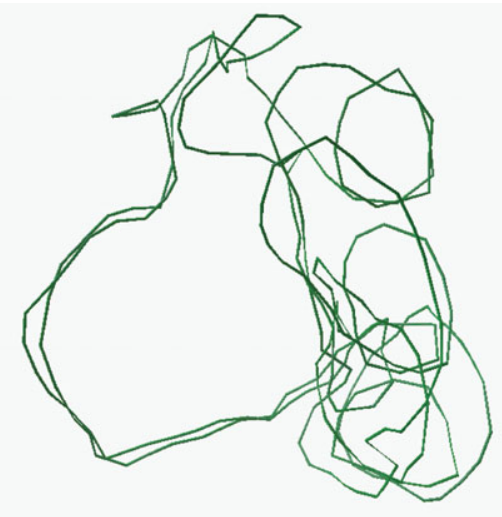

(d)

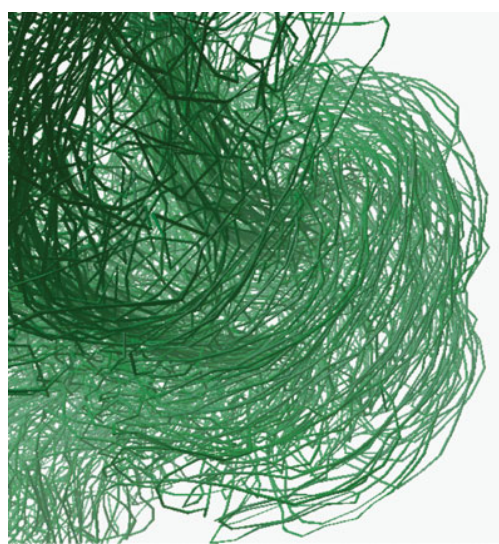

FIGURE 3. (Colour online) Evolution of the initial seed superfluid vortex ring into an expanding cloud. Time increases from $(a)$ towards $(d)$ as follows: $(a) t_{a}=1.444 \times 10^{-2} \mathrm{~s}$, (b) $t_{b}=2.982 \times 10^{-2} \mathrm{~s},(c) t_{c}=7.073 \times 10^{-2} \mathrm{~s}$ and $(d) t_{d}=7.944 \times 10^{-2} \mathrm{~s}$ (detail).

energies in isolation. Indeed, as we show next, the physical mechanisms responsible for the expansion of the superfluid vortex cloud verify this conclusion.

\subsection{Physics of superfluid vortex tangle growth}

Figure 3 shows the evolution of the seed superfluid vortex ring. Using the formula (Barenghi et al. 1997) $V_{O G}=(\kappa / 2 \theta) \ln \left(\theta / 2 \pi a_{0}\right)$, where $a_{0} \approx 10^{-8} \mathrm{~cm}$ is the superfluid vortex core for ${ }^{4} \mathrm{He}$, we compute the critical velocity of the OstermeierGlaberson instability of the smallest resolvable scales along the quantized vortices, $V_{O G}=1.725 \mathrm{~cm} \mathrm{~s}^{-1}$. Since the intensity of normal-fluid turbulence $V_{n}=28.52 \mathrm{~cm} \mathrm{~s}^{-1}$ is much higher than this velocity, we expect the instability to be active. Indeed, the initial ring becomes unstable and, remarkably, the newly added length is folded back onto the original ring creating a new ring of double circulation strength (figure $3 a$ ). Subsequent instabilities (figure $3 b$ ) do not follow this pattern, and subsequent tangle configurations (figure $3 c$ ) do not show, within the cloud, the superfluid bundles observed in the kinematic computations of Kivotides (2006) and Kivotides \& Wilkin (2009). This could be the case because, as discussed above and in Kivotides (2007a), 

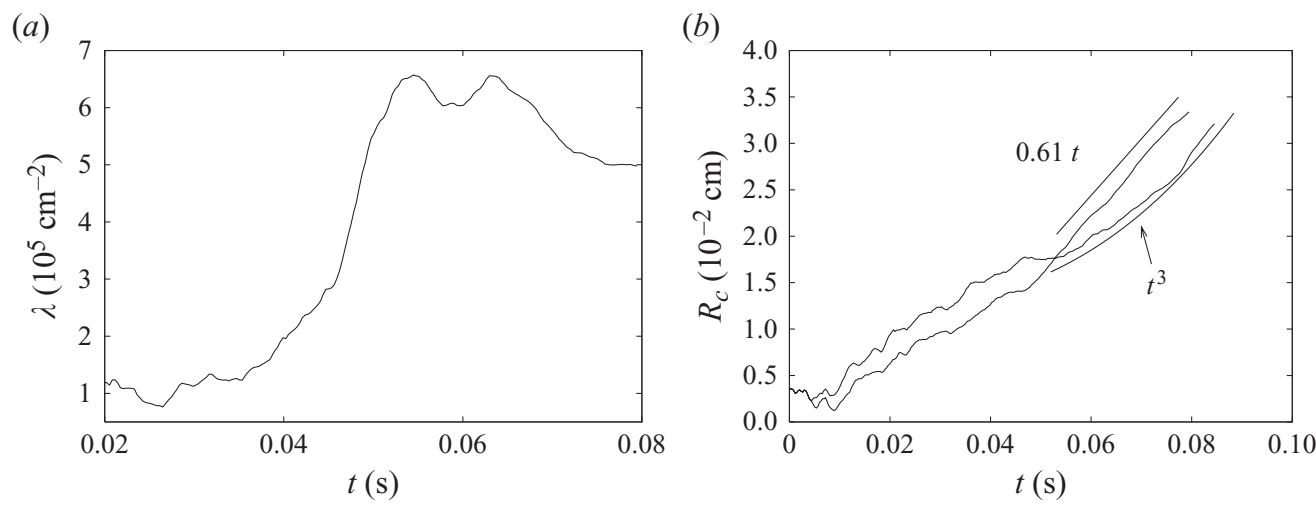

Figure 4. (a) Superfluid vortex density, $\lambda$, versus time, $t$. (b) Superfluid vortex cloud radius, $R_{c}$, versus time, $t$. The line fitted with the power law indicates $R_{c}$ evolution in an accompanying kinematic computation. Both $R_{c}$ curves present a short-lived plateau around $t \approx 5 \times 10^{-2} \mathrm{~s}$ that signifies the end of an initial linear growth transient. The plateau corresponds to the actual formation of the superfluid cloud, i.e. the homogenization of its inner volume before the beginning of its steady-state expansion. Indeed, $\lambda$ grows rapidly during the $R_{c}$ plateau period before it becomes stabilized. Notably, the computation stops long before the cloud radius reaches half of the box size $l_{b}\left(l_{b}=10^{-1} \mathrm{~cm}\right)$.

the generated superfluid vorticity damps the normal-fluid structure responsible for its generation and subsequent coherence. Figure $3(c)$ shows the whole superfluid vortex cloud, while figure $3(d)$ provides a detailed view of the superfluid tangle at a typical location within the interface layer between the bulk of the cloud and the normal-fluid turbulence bath. The vortex structure in figure $3(d)$ resembles the spiral structure of Kivotides \& Wilkin (2009) (figure 2c) which is the signature of the interaction between normal-fluid vorticity tubes of the outer (with respect to the cloud) turbulence and superfluid vortices. We compute the radius $R_{c}$ of the cloud with the formula

$$
R_{c}^{2}=\frac{1}{N_{v}} \sum_{i=1}^{N_{v}}\left(\boldsymbol{X}_{s}^{i}-\boldsymbol{R}_{c m}\right)^{2},
$$

where the cloud's centre $\boldsymbol{R}_{c m}$ is given by

$$
\boldsymbol{R}_{c m}=\frac{1}{N_{v}} \sum_{i=1}^{N_{v}} \boldsymbol{X}_{s}^{i} .
$$

The evolution of $R_{c}$ with time is shown in figure 4(b). Employing as reference value the volume of the sphere with radius $R_{c}$, we compute the vortex line density shown in figure 4(a). Regarding $R_{c}$, there is an initial linear growth transient that ends in a short-lived plateau around $t \approx 5 \times 10^{-2} \mathrm{~s}$. The starting linear growth is reminiscent of a similar observation in classical fluids (Tulin \& Wu 1997). The plateau signifies the actual formation of the superfluid cloud, i.e. the homogenization of its inner volume before the beginning of its steady-state expansion. Indeed, $\lambda$ grows rapidly during the $R_{c}$ plateau period before it becomes stabilized (figure $4 a$ ). Notably, up to the plateau region, the $R_{c}$ evolution is similar to the corresponding one in an accompanying kinematic computation (dashed line in figure $4 b$ ). Subsequently, the self-consistent $R_{c}$ grows linearly with time, whilst the kinematic one grows according to a power law $\left(R_{c} \sim t^{3}\right)$. Certainly, the power-law growth in the kinematic case has to do with lack 


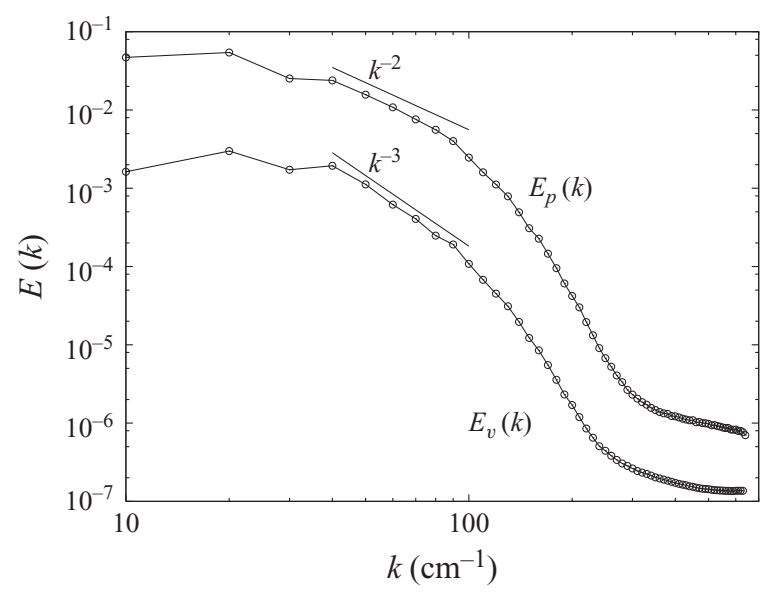

FIGURE 5. Energy (lower curve) and pressure spectra (upper curve) of the superfluid vortex cloud. The $k^{-3}$ energy and $k^{-2}$ pressure scalings coincide in wavenumber space. Moreover, their $k$ support corresponds exactly to the $k$ regime characterized by the Kolmogorov $k^{-5 / 3}$ scaling in the pure normal-fluid turbulence region (figure $1 a$ ).

of any asymmetry between the inner and outer superfluid cloud domains as far as normal-fluid turbulence is concerned. Since this asymmetry is induced by the reaction of the superfluid vortices on the normal-fluid flow, the power-law growth rate is an artefact of the kinematic approximation that cannot be observed experimentally. On the other hand, the linear growth of $R_{c}$ in the steady-state fully coupled case can be explained as follows: the tangle grows via the spiral vortex forming mechanism of normal vortex tube-superfluid vortex interaction (Kivotides \& Wilkin 2009) that continuously takes place in the interface layer. Indeed, the superfluid energy spectrum of figure 5 (lower curve) shows the characteristic $k^{-3}$ scaling of this mechanism. Since, the intensity of the spiral-forming mechanism scales with the normal-fluid velocity (Kivotides \& Wilkin 2009), it is plausible to expect (on dimensional analysis grounds) that $\mathrm{d} R_{c} / \mathrm{d} t$ should scale with the intensity of turbulence ahead of the cloud $V_{n}$. The fact that the Kolmogorov spectrum of the latter (figure 1a) and the $k^{-3}$ scaling of the superfluid energy spectrum (figure 5) have the same support in $k$ space is also consistent with this suggestion (i.e. inertial range motions (characterized by $V_{n}$ ) excite the spirals that drive cloud expansion). Accordingly, $\mathrm{d} R_{c} / \mathrm{d} t=\beta V_{n}$, with the results suggesting a weak coupling $\beta=0.0214$. Figure 5 shows also the spectrum of the scalar pressure field $p_{s} / \rho_{s}$ whose governing equation is

$$
\rho_{s}^{-1} \nabla^{2} p_{s}=\omega_{s}^{2}-s_{s}^{2} .
$$

Here, $\omega_{s}^{2}$ is the superfluid enstrophy and $s_{s}^{2}$ is the superfluid total strain. The observed $k^{-2}$ scaling overlaps in $k$ space with the $k^{-3}$ energy scaling. It agrees with the prediction of Kivotides et al. (2001c) that, based on the implications of the extreme localization of superfluid vorticity on the Fourier transform of the pressure field, proposed the computed $k^{-2}$ pressure scaling as a quantum signature of superfluid turbulence. Moreover, again due to the extreme localization of superfluid vorticity $\omega_{s}(\boldsymbol{x})$, its Fourier transform $\hat{\omega}_{s}(\boldsymbol{k})$ is, for $k \ll 1 / a_{0}(k=|\boldsymbol{k}|)$, a very slowly varying (in wavenumber space) function, thus $\left|\hat{\omega}_{s}(\boldsymbol{k})\right|^{2} \sim k^{0}$. Hence, since its spectrum $E_{\omega_{s}}(k)$ is defined as $\int_{0}^{\infty} E_{\omega_{s}}(k) \mathrm{d} k=\iiint\left|\hat{\omega}_{s}(\boldsymbol{k})\right|^{2} \mathrm{~d}^{3} \mathbf{k}$, it follows that $E_{\omega_{s}}(k) \sim k^{2}$. 
Notably, the tangle configurations in figure 3 do not show any small vortex loops emitted by the superfluid turbulent region. Such small loops appear in pure superfluid (Barenghi \& Samuels 2002) or thermal superfluid computations with kinematically prescribed zero normal-fluid flow (Tsubota, Araki \& Vinen 2003). In our case, however, the normal-fluid inertia is large and the accompanying strong smoothing mutual friction effects do not allow the highly corrugated quantized vortex configurations that, via reconnections, lead to very small vortex loops. Notably, Nemirovskii has recently developed a theory of superfluid vortex cloud expansion in terms of a diffusion equation for the vortex-line density (Nemirovskii 2010). This theory is useful in the context of the pure superfluid Barenghi \& Samuels (2002) or thermal superfluid with zero normal-fluid inertia (Tsubota et al. 2003) cases discussed above, but it is not applicable in our case since cloud expansion here is driven by spiral structure forming instabilities that occur at the interface between the vortex cloud and highly energetic free normal-fluid turbulence. This physical mechanism is not included in the above diffusion theory.

Finally, it is useful to compare the rate of cloud growth due to the above mutualfriction-driven mechanism with a corresponding one due solely to the dispersion properties of normal-fluid turbulence. Consider, for example, any two markers on the initial superfluid ring separated by distance $\Delta X$ equal to the ring diameter, i.e. $\Delta X(0)=0.007 \mathrm{~cm}$. Then according to Richardson's law for the relative diffusion of two particles (Davidson 2004), $\Delta X(t) \sim \Delta X(0)+\sqrt{\epsilon} t^{3 / 2}$, thus $\Delta X\left(t_{f}\right) \sim 27 \mathrm{~cm}$ which is orders of magnitude larger than the computational domain size. Thus, in agreement with our analysis above, the growth of the cloud is not fuelled by turbulent dispersion.

\subsection{Fractal dimension of superfluid vortex tangle}

There are deep connections between the fractal dimension of quasi-singular vorticity and energy spectrum scaling in turbulent flows (Chorin 1994). Thus, following Kivotides, Barenghi \& Samuels (2001b), it is instructive to determine the fractal dimension, $d_{f}$, of the superfluid tangle. This we perform with the standard box counting method (Vassilicos \& Brasseur 1996). The algorithm computes $N(\delta)$, the minimum number of boxes of size $\delta$ needed to cover all points produced by the discretization of superfluid vortices. Here, we note that the largest distance between two consecutive points along the filaments is at most 1.65 times greater than the smallest distance. With these data, we construct a $\log -\log$ graph of $N(\delta)$ versus $\delta$. If a fractal dimension exists, then for an adequately large range of $\delta$ values, a slope should be visible on the graph defining the fractal dimension $d_{f}$ : $\ln \left(N(\delta) / N\left(\delta_{\text {min }}\right)\right)=-d_{f} \ln \left(\delta / \delta_{\min }\right)$. Figure $6(a)$, shows $d_{f}$ as a function of the vortexline density. Notably, $d_{f}$ saturates at $\left\langle d_{f}\right\rangle \approx 1.652$. Figure $6(b)$ shows a definite fractal scaling in the regime $0.001<\delta<0.03$ which includes the range of scales exhibiting the $k^{-3}$ scaling in the energy spectrum and extends all the way to the intervortex spacing. For comparison, Kivotides \& Leonard (2004) found $d_{f}=1.8$ for the centrelines of vortex tubes in classical turbulence. The fact that the Kolmogorov spectrum corresponds to a larger fractal dimension than the present $k^{-3}$ spectrum reflects the relative simplicity of the dynamics governing the generation and evolution of superfluid cloud vorticity in comparison with classical vortex dynamics. The latter encodes strongly nonlinear processes in a self-interacting field, whilst the former originates in mutual friction action and related instabilities that lead to much simpler geometrical vorticity patterns. 
(a)

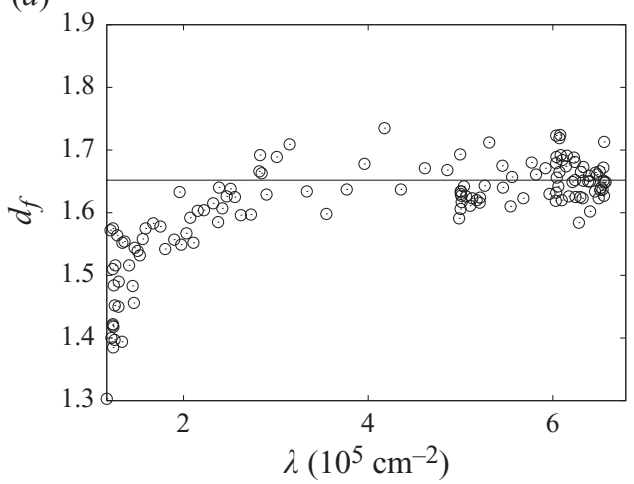

(b)

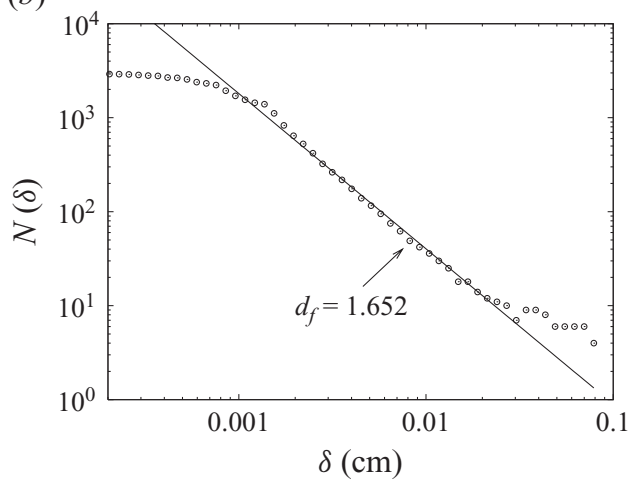

Figure 6. (a) Fractal dimension, $d_{f}$, versus superfluid vortex line density, $\lambda$. Evidently, $d_{f}$ saturates at average value $\left\langle d_{f}\right\rangle \approx 1.652$. $(b)$ Number, $N$, of boxes versus box size, $\delta$, and determination of the fractal dimension, $d_{f}$, of the superfluid vortex tangle at $t=0.0674 \mathrm{~s}$ when $\lambda \approx 0.6096 \times 10^{6} \mathrm{~cm}^{-2}$.

\section{Conclusion}

We have formulated a self-consistent model of thermal superfluid dynamics. By employing the model, we have analysed the problem of superfluid vorticity cloud propagation in normal-fluid turbulence. We have shown that superfluid cloud expansion is driven by pattern-forming superfluid vortex instabilities taking place in the interface layer between the cloud's bulk and the outer undisturbed normalfluid turbulence. The radius of the cloud increases linearly with time. Mutual friction transfers energy from the normal-fluid turbulence to the superfluid cloud, whilst damping the smallest normal-fluid turbulence motions. This damping action is much weaker than viscous dissipation effects in a corresponding pure normal-fluid turbulence. The energy spectrum of superfluid turbulence presents the $k^{-3}$ scaling of Kivotides \& Wilkin (2009) that characterizes the spiral superfluid vorticity patterns of normal vortex tube-superfluid vortex interactions. The corresponding $k^{-2}$ pressure spectrum indicates (in the fashion of Kivotides et al. 2001c) the singular nature of superfluid vorticity. These two scalings coincide in wavenumber space with the Kolmogorov regime in the normal-fluid turbulence. We compute a fractal dimension $d_{f} \approx 1.652$ for superfluid vorticity. This value is smaller that the corresponding $d_{f}=1.8$ fractal dimension of vortex tube centrelines in classical turbulence (Kivotides \& Leonard 2004). This is because, the mutual-friction-dominated superfluid vorticity dynamics in the present case are much simpler than the strongly nonlinear classical vortex dynamics. The latter lead to much more vigorous vortex stretching and folding mechanisms that result in analogously complex vorticity contours (Kivotides \& Leonard 2004).

A crucial question in statistical theories of thermal superfluid turbulence (Peralta et al. 2006, 2008; Andersson et al. 2007; Sidery et al. 2008) is whether the average (macroscopic) effects of mutual friction could be modelled by an isotropic (Gorter and Mellink) or rectilinear-array-type (Hall \& Vinen 1956) laws. The present results suggest that (in homogeneous isotropic turbulence) neither approach is absolutely correct. Instead it appears that, for a particular thermal superfluid scale, the normalfluid eddies first generate ordered, spiral-like superfluid structures while themselves being damped in the process. The damping of the generating normal-fluid eddies would eventually allow the superfluid structures to relax (always at the particular 
scale of interest) to an isotropic state. The time scales of this relaxation, as well as a more detailed analysis of these processes requires further specially targeted investigations. In this context, it is worth noting that our results could offer a microscopic understanding of the physics of superfluid vortex tangle patches of laminar-turbulent transition in neutron stars as discussed by Peralta et al. 2006.

In contrast to kinematic computations (Barenghi et al. 1997; Kivotides 2006), the results do not show that a turbulent normal-fluid with a Kolmogorov energy spectrum induces superfluid vortex bundles in the superfluid. Since it has been demonstrated, in both classical (Kivotides \& Leonard 2003) and superfluid (Kivotides 2006) contexts, that systems of vortex bundles present a $k^{-5 / 3}$ energy spectrum scaling, the absence of vortex bundles in the present results is consistent with the observed $k^{-3}$ scaling. Moreover, in contrast to claims made based on the kinematic computation of Morris, Koplik \& Rouson (2008), we do not see any evidence of 'vortex locking' in our results. In fact, as shown in Kivotides \& Wilkin (2009), the alignment between normal-fluid and superfluid vorticities in the above calculation is not the dominant dynamical process in normal-fluid/superfluid vortex interactions. The most important effect (observed also here) is the formation of superfluid vortices on planes normal to the normal-fluid vortices and the associated $k^{-3}$ energy spectrum scaling. Notably, our direct numerical calculation indicates that the reaction of the superfluid on the normal fluid is important and that mutual-friction-induced damping of normal-fluid vortex structures is a crucial dynamical factor. The latter is also evident in the different superfluid vortex cloud growth rates found for the kinematic and fully coupled cases of figure $4(b)$.

A desirable future development would be the extension of the present approach to the problem of the fully developed thermal superfluid turbulence. In particular, it is important to investigate whether the superfluid tangle could acquire any dynamically important structure on the large scales that precede in $k$ space the present $k^{-3}$ spectrum. Notably, the complexity of the current computations does not allow us, at present, to perform a systematic study of the effect of temperature variations on the conclusions. However, in simpler cases, where such a study was practically feasible (Kivotides \& Wilkin 2008), increasing temperatures resulted in notably smoother superfluid vortex contours (i.e. augmented Kelvin wave damping) without qualitatively altering the conclusions.

Valuable discussions and correspondence with L. Wilkin, R. Hanninen and G. Volovik are gratefully acknowledged.

\section{REFERENCES}

Andersson, N., Sidery, T. \& Comer, G. L. 2007 Superfluid neutron star turbulence. Mon. Not. R. Astron. Soc. 381, 747-756.

Barenghi, C. F., Donnelly, R. J. \& Vinen, W. F. 1983 Friction on quantized vortices in Helium II. A review. J. Low Temp. Phys. 52, 189-247.

Barenghi, C. F. \& Niemela, J. J. (Ed.) 2009 Quantum turbulence, Special Issue: J. Low Temp. Phys. 156.

Barenghi, C. F. \& Samuels, D. C. 2002 Evaporation of a packet of quantized vorticity. Phys. Rev. Lett. 89, 155302-155306.

Barenghi, C. F., Samuels, D. C., Bauer, G. H. \& Donnelly, R. J. 1997 Superfluid vortex lines in a model of turbulent flow. Phys. Fluids 9, 2631-2644.

Bewley, G. P., Lathrop, D. P. \& Sreenivasan, K. R. 2006 Superfluid helium: visualization of quantized vortices. Nature 44, 588. 
Chorin, A. 1994 Vorticity and Turbulence. Springer.

DAvidson, P. A. 2004 Turbulence. Oxford University Press.

Finne, A. P., Eltsov, V. B., Hanninen, R., Kopnin, N. B., Kopu, J., Krusius, M., Tsubota, M. \& Volovik, G. E. 2006 Dynamics of vortices and interfaces in superfluid ${ }^{3}$ He. Rep. Prog. Phys. 69, 3157-3230.

Fisher, S. N. \& PicketT, G. R. 2008 Quantum turbulence in superfluid ${ }^{3} \mathrm{He}$ at very low temperatures. Prog. Low Temp. Phys. 16, 147-194.

Gardiner, C. 2004 Handbook of Stochastic Methods: for Physics, Chemistry and the Natural Sciences. Springer.

Griffin, A., Kinuni, T. \& Zaremba, E. 2009 Bose-Condensed Gases at Finite Temperatures. Cambridge University Press.

Guo, W., Wright, J. D., Cahn, S. B., Nikkel, J. A. \& McKinsey, D. N. 2009 Metastable helium molecules as tracers in superfluid ${ }^{4}$ He. Phys. Rev. Lett. 102, 235301-235305.

Hall, H. E. \& Vinen, W. F. 1956 The rotation of liquid Helium II. Part II. The theory of mutual friction in uniformly rotating Helium II. Proc. R. Soc. Lond. A 238, 215-234.

Henderson, K. L. \& Barenghi, C. F. 2004 Superfluid Couette flow in an enclosed annulus. Theor. Comput. Fluid Dyn. 18, 183-196.

Idowu, O. C., Kivotides, D., Barenghi, C. F. \& Samuels, D. C. 2000 Equation for self-consistent superfluid vortex line dynamics. J. Low Temp. Phys. 120, 269-280.

JaCkson, B., Barenghi, C. F. \& Proukakis, N. P. 2007 Matter wave solitons at finite temperatures. J. Low Temp. Phys. 148, 387-391.

Kivotides, D. 2006 Coherent structure formation in turbulent thermal superfluids. Phys. Rev. Lett. 96, $17530-17534$.

Kivotides, D. 2007a Relaxation of superfluid vortex bundles via energy transfer to the normal fluid. Phys. Rev. B76, 054503-054515.

Kivotides, D. $2007 b$ Superfluid contributions to general turbulence theory. J. Low Temp. Phys. 148, 287-291.

Kivotides, D. 2008 a Normal-fluid velocity measurement and superfluid vortex detection in thermal counterflow turbulence. Phys. Rev. B 78, 224501-224505.

Kivotides, D. $2008 b$ Motion of a spherical solid particle in thermal counterflow turbulence. Phys. Rev. B 77, 174508-174513.

Kivotides, D., Barenghi, C. F. \& Samuels, D. C. 2001 a Superfluid vortex reconnections at finite temperature. Europhys. Lett. 54, 774-778.

Kivotides, D., Barenghi, C. F. \& Samuels, D. C. $2001 b$ Fractal dimension of superfluid turbulence. Phys. Rev. Lett. 87, 155301-155305.

Kivotides, D., Vassilicos, J. C., Barenghi, C. F., Khan, M. A. I \& Samuels, D. C. 2001c Quantum signature of superfluid turbulence. Phys. Rev. Lett. 87, 275302-275306.

Kivotides, D., Barenghi, C. F. \& Sergeev, Y. A. $2008 a$ Interactions between particles and quantized vortices in superfluid helium. Phys. Rev. B 77, 014527-014540.

Kivotides, D., Sergeev, Y. A. \& Barenghi, C. F. $2008 b$ Dynamics of solid particles in a tangle of superfluid vortices at low temperatures. Phys. Fluids 20, 055105-055115.

Kivotides, D. \& Leonard, A. 2003 Quantized turbulence physics. Phys. Rev. Lett. 90, 234503234507.

Kivotides, D. \& Leonard, A. 2004 Geometrical physics of the many vortex filament problem. Europhys. Lett. 66, 69-75.

Kivotides, D. \& Wilkin, S. L. 2008 Collisions of solid particles with vortex rings in superfluid helium. J. Fluid Mech. 605, 367-387.

Kivotides, D. \& Wilkin, S. L. 2009 Elementary vortex processes in thermal superfluid turbulence. J. Low Temp. Phys. 156, 163-181.

Koplik, J. \& Levine, H. 1993 Vortex reconnection in superfluid helium. Phys. Rev. Lett. 71, 1375-1379.

Leggett, A. J. 2006 Quantum Liquids: Bose Condensation and Cooper Pairing in Condensed-Matter Systems. Oxford University Press.

Lundgren, T. S. 2003 Linearly forced isotropic turbulence. Center for Turbulence Research, Annual Research Briefs, p. 461.

Maurer, J. \& TAbeling, P. 1998 Local investigation of superfluid turbulence. Europhys. Lett. 43, 29-34. 
Melatos, A. \& Peralta, C. 2010 Gravitational radiation from hydrodynamic turbulence in a differentially rotating neutron star. Astrophys. J. 709, 77-87.

Mine, M., Okumura, M., Sunaga, T. \& Yamanaka, Y. 2007 Quantum field theoretical description of dynamical instability of trapped Bose-Einstein condensates. J. Low Temp. Phys. 148, 331-336.

Morris, K., Koplik, J. \& Rouson, D. W. I. 2008 Vortex locking in direct numerical simulations of quantum turbulence. Phys. Rev. Lett. 101, 015301-015305.

NemirovskiI, S. K. 2010 Diffusion of inhomogeneous vortex tangle and decay of superfluid turbulence. Phys. Rev. B 81, 064512-064522.

Parker, N. G., Proukakis, N. P., Barenghi, C. F. \& Adams, C. S. 2004 Controlled vortex sound interaction in atomic Bose-Einstein condensate. Phys. Rev. Lett. 92, 160403-160407.

Peralta, C., Melatos, A., Giacobello, M. \& Ooi, A. 2005 Global three-dimensional flow of a neutron superfluid in a spherical shell in a neutron star. Astrophys. J. 635, 1224-1232.

Peralta, C., Melatos, A., Giacobello, M. \& Ooi, A. 2006 Transitions between turbulent and laminar superfluid vorticity states in the outer core of a neutron star. Astrophys. J. 651, 1079-1091.

Peralta, C., Melatos, A., Giacobello, M. \& Ooi, A. 2008 Superfluid spherical Couette flow. J. Fluid Mech. 609, 221-274.

Pitaevskit, L. \& Stringari, S. 2003 Bose-Einstein Condensation. Oxford University Press.

Samuels, D. C. \& Kivotides, D. 1999 A damping length scale for superfluid turbulence. Phys. Rev. Lett. 83, 5306-5310.

Schwarz, K. W. 1995 Three-dimensional vortex dynamics in superfluid ${ }^{4} \mathrm{He}$ : line-line and lineboundary interactions. Phys. Rev. B 31, 5782-5804.

Sidery, T., Andersson, N. \& Comer, G. L. 2008 Waves and instabilities in dissipative rotating superfluid neutron stars. Mon. Not. R. Astron. Soc. 385, 335-348.

Skrbek, L. \& Vinen, W. F. 2008 The use of vibrating structures in the study of quantum turbulence. Prog. Low Temp. Phys. 16, 195-246.

Sonin, E. B. 1997 Magnus force in superfluids and superconductors. Phys. Rev. B 55, 485-501.

Stalp, S. R., Skrbek, L. \& Donnelly, R. J. 1999 Decay of grid turbulence in a finite channel. Phys. Rev. Lett. 82, 4831-4835.

STONE, M. 2000 Iordanskii force and the gravitational Aharonov-Bohm effect for a moving vortex. Phys. Rev. B 61, 11780-11786.

Thouless, D. J., Ao, P. \& Niu, Q. 1996 Transverse force on a quantized vortex in a superfluid. Phys. Rev. Lett. 76, 3758-3762.

Tsubota, M., Araki, T. \& Vinen, W. F. 2003 Diffusion of an inhomogeneous vortex tangle. Physica B 329, 224-225.

Tsubota, M. \& Kobayashi, M. 2008 Energy spectra of quantum turbulence. Prog. Low Temp. Phys. $16,1-43$.

Tulin, M. P. \& Wu, J. 1977 Additive effects on free turbulent flows. Phys. Fluids 20, S109-S113.

VAssilicos, J. C. \& Brasseur, J. G. 1996 Self-similar spiral flow structure in low-Reynolds-number isotropic and decaying turbulence. Phys. Rev. E 54, 467-485.

VINEN, W. F. 2000 Classical character of turbulence in a quantum liquid. Phys. Rev. B 61, 1410-1420.

Vinen, W. F. \& Niemela, J. J. 2002 Quantum turbulence. J. Low Temp. Phys. 128, 167-231.

VoLOVIK, G. E. 1998 Vortex versus spinning string: Iordanskii force and gravitational AharonovBohm effect. J. Exp. Theor. Phys. Lett. 67, 881-886.

WeXLer, C. 1997 Magnus and Iordanskii forces in superfluids. Phys. Rev. Lett. 79, 1321-1325.

Wilkin, S. L., Barenghi, C. F. \& Shukurov, A. 2007 Magnetic structures produced by the small-scale dynamo. Phys. Rev. Lett. 99, 134501-134505.

Wilson, J. R. \& Mathews, G. J. 2007 Relativistic Numerical Hydrodynamics. Cambridge University Press.

Zhang, T. \& VAN ScIVER, S. W. 2005 The motion of micron-sized particles in He II counterflow as observed by the PIV technique. J. Low Temp. Phys. 138, 865-870. 